\title{
What can polysemy tell us about theories of explanation?
}

\author{
Maria Şerban ${ }^{1,2}$
}

Received: 19 February 2015 / Accepted: 7 March 2016 / Published online: 30 March 2016

(C) The Author(s) 2016. This article is published with open access at Springerlink.com

\begin{abstract}
Philosophical accounts of scientific explanation are broadly divided into ontic and epistemic views. This paper explores the idea that the lexical ambiguity of the verb to explain and its nominalisation supports an ontic conception of explanation (Salmon 1989; Craver 2007). I analyse one argument which challenges this strategy by criticising the claim that explanatory talk is lexically ambiguous (Wright, European Journal of Philosophy of Science 2(3), $375-394,2012)$. I propose that the linguistic mechanism of transfer of meaning (Nunberg, Journal of Semantics 12(2), 109-132, 1995) provides a better account of the lexical alternations that figure in the systematic polysemy of explanatory talk, and evaluate the implications of this proposal for the debate between ontic and epistemic conceptions of scientific explanation.
\end{abstract}

Keywords Explanation · Ontic $\cdot$ Epistemic $\cdot$ Ambiguity $\cdot$ Polysemy $\cdot$ Predicate transfer

\section{Introduction}

Explanation, it has been often claimed, is one of the distinctive virtues of theoretical science, which aspires to go beyond the mere description of observed regularities and generate insights which afford us a better intellectual grasp of the phenomena of

Maria Şerban

M.Serban@1se.ac.uk

1 London School of Economics and Political Science, London, UK

2 Centre for Philosophy of Natural and Social Science, London, UK 
interest, i.e., an understanding of why things are as they are. The idea that explaining something implies seeking to understand it and make it intelligible seems to support the view that explanation is primarily an epistemic affair and that scientific explanations are among our most important epistemic achievements. In line with this line of thought, the primary task of a theory of (scientific) explanation is to answer the epistemological question of what sort of knowledge we gain through explanation. Or, otherwise put, what exactly is our epistemic gain of having an explanation? A related but different way of approaching the problem of scientific explanation is to ask in virtue of what objective fact or property does an explanatory relation hold in the first place.

Those who are inclined to take the former approach typically defend an epistemic view of scientific explanation. According to these philosophers, scientific explanation is an activity internal to a certain system or body of scientific knowledge. That is, factors internal to a particular system of knowledge and the relationships between them determine when something counts as a kosher explanation which can then be evaluated in terms of its cognitive success, empirical adequacy, etc. On this picture, the world contributes to our explanatory activities only insofar as it guarantees the truth of the explanatory statements. ${ }^{1}$ On the other side of the debate, proponents of an ontic or metaphysical view of scientific explanation hold that the explanatory link between the explanandum and the explanans is grounded in some objective relation which holds between the phenomena/events described (or otherwise represented) by the two component statements of an explanation. Subsequently, on this view, the task of a theory of explanation is to attempt to clarify the nature of this objective relation.

The debate between ontic and epistemic views of scientific explanation constitutes the background for many arguments currently discussed in the philosophy of explanation. Even if the field has seen a more recent turn towards a case-study based style of argumentation which seeks to identify the norms that govern the construction and evaluation of particular modes of explanation used in different sciences, the ontic vs epistemic divide continues to inform the proposed philosophical models of scientific explanation (e.g., Craver 2007; Illari 2013; Sheredos 2015; van Eck 2015; Wright 2015). This is partly due to the fact that this debate has typically been said to determine the form that a philosophical analysis of the nature of scientific explanation should take in the first place. On the ontic view of scientific explanation, the theorising efforts should focus on clarifying the objective relationship which grounds explanatory claims, whereas on the epistemic view, one should prioritise the task of showing how explanatory knowledge differs from other types of knowledge.

This paper analyses one argument strategy which has been used by defenders of the ontic view of scientific explanation to support the priority of the metaphysical approach over the epistemological one. The central step in this argument is the assumption that the verb to explain and its nominalisation explanation are lexically

\footnotetext{
${ }^{1}$ This description of the epistemic view of scientific explanation is close to Kim's (1994) analysis of 'explanatory internalism', according to which 'apart from the determination of the truth of the explanatory premisses, the evaluation of an explanation is a purely internal affair, in that the objects involved are statements and they are examined in respect of their form and mutual logical relationships' (p. 55).
} 
ambiguous. This is then taken to licence the inference that the ontic conception of explanation (OC) is logically prior to the epistemic conception (EC). In order to evaluate this argument, I will focus on the causal/mechanistic view of scientific explanation. There are two main reasons for choosing this account over other candidates. Firstly, causation has been one of the strongest traditional candidates for grounding the relation of explanatory relevance. In consequence, most proponents of the ontic view of explanation defend one or other version of the causal view of explanation. Secondly, the fact that the causal/mechanistic framework allows the formulation of both epistemic and ontic conceptions of explanation facilitates the comparison between the two stances and their relative advantages and limitations. ${ }^{2}$

The structure of the paper divides into three distinct parts. I begin in Section 2 by discussing a semantically based argument put forward in support of the ontic view of causal/mechanistic explanation (cf. Salmon 1989). According to this line of reasoning, relying on the lexical ambiguity of the term explanation, one can show that among the multiple senses of explanation there is at least one which corresponds to the ontic conception of explanation and, in addition, that this 'ontic' sense of explanation is conceptually basic. Section 3 analyses the linguistic arguments advanced by Cory D. Wright (2012) against the hypothesis that explanation is lexically ambiguous in the first place. In response, Section 4 aims to show that the linguistic phenomena that defenders of OC have in mind are better diagnosed as cases of systematic polysemy which is a sub-species of lexical ambiguity. More specifically, I contend that the linguistic process of transfer of meaning (Nunberg 1995) is better suited to account for the lexical alternations observed in the uses of the verb to explain and its nominalisation. In the final section, I assess the consequences of this proposal for the debate between epistemic and ontic conceptions of scientific explanation.

\section{A semantic argument for ontic explanations}

The ontic conception of explanation has been promoted primarily by theorists who maintain that scientific explanation is broadly causal explanation (e.g., Lewis 1986; Salmon 1984, 1989; Glennan 1996, 2002; Craver 2007, Machamer et al. 2000 etc.). For the purposes of this paper, I will focus on the mechanistic version of the causal view of scientific explanation. According to this view, to explain a given event/phenomenon or regularity is to show how it fits in the causal structure of the world, or differently put, to exhibit the causal mechanisms that are responsible for its occurrence. The generic notion of a mechanism, on which virtually all defenders of this view seem to have converged, is that of a complex hierarchical system comprising a series of component parts whose operations are organised so that they

\footnotetext{
${ }^{2}$ In the causal/mechanistic tradition, William Bechtel and Cory Wright are some of the most prominent defenders of the epistemic conception of mechanistic explanation (Bechtel and Richardson 1993/2010; Bechtel and Abrahamsen 2006; Wright and Bechtel 2007; Bechtel 2008; Wright 2012 etc.). Proponents of the ontic conception of scientific explanation include Wesley Salmon (1984, 1989), Stuart Glennan (1996, 2002), and Carl Craver (2007, 2012).
} 
generate the phenomenon/regularity that is to be explained (cf. Wright 2012). Thus, the ontic conception claims that scientific explanations reveal the mechanisms which are responsible for the events and/or causal regularities of interest.

The ontic formulation of the mechanistic view of explanation comes in both radical and more moderate forms. ${ }^{3}$ According to the radical interpretation, explanations are simply the actual (or real) mechanisms operating in the world and producing the phenomena one takes as the target of explanation. This reading is not only explicitly stated in Craver (2012), but it also seems to support the claim that the problem of representation in science is orthogonal to the explanation problem. One can be committed to an account of the latter issue (identifying explanations with mechanisms, for instance) without having any settled view of what counts as a good representation or why abstract or idealized representational models are sometimes deemed to have explanatory force. The moderate reading is present in both Salmon's (1989) and Craver's (2007) writings and it states that representational models of actual mechanisms can be seen as explanations. While the moderate interpretation allows for a more substantial common ground between the ontic and the epistemic conceptions (by recognizing the explanatory value of epistemic representations), it still forces defenders of the epistemic conception in accepting a questionable priority claim with respect to the ontic perspective. ${ }^{4}$

There are two primary motivations that seem to drive the ontic version of the causal/mechanistic view of scientific explanation. First, the view promises to offer an alternative to the covering-law model of scientific explanation (Hempel and Oppenheim 1948) which construes explanations as arguments and cashes out the explanatory relevance relation in logico-linguistic terms (Salmon 1984; Lewis 1986). That is, the causal/mechanistic model arguably overcomes the challenges faced by the Hempelian model of explanation by postulating that the relevant explanatory link depends on the causal dependence relations holding between certain parts of the (external) world rather than on the ways in which we describe or represent these relations. Second, it has been argued that the ontic conception secures the objectivity of scientific explanations by grounding them in causal dependency relations encountered in the world itself rather than in the epistemic practices of particular scientific communities (e.g., Glennan 2002, 2005; Craver 2012).

Taken as a general research strategy, the mechanistic framework seems to be neutral between an epistemic and an ontic view of scientific explanation. However, in light of the motivations sketched above, an increasing number of mechanists seem to prefer the ontic version of the causal/mechanistic model of scientific explanation. For instance, Glennan (2002, p. 348) writes that "while it is sometimes the case that a description of the parts of the mechanism will entail a description of the

\footnotetext{
${ }^{3}$ These are not always explicitly distinguished by proponents of the ontic conception. For a recent analysis that looks more in depth at the different versions of the ontic conception, see Wright (2015).

${ }^{4}$ Illari (2013), Sheredos (2015) and van Eck (2015) have convincingly argued that the ontic priority claim is not strongly supported by either the conceptual or the empirical arguments offered in Salmon (1989), Glennan (2005), Craver $(2007,2012)$ or other defenders of the ontic conception of mechanistic explanation.
} 
mechanism's outward behavior, the explanation lies not in the logical relationship between these descriptions but in the causal relationships between the parts of the mechanisms that produce the behavior described.' More recently, Craver has pointed out that the component entities and activities of a mechanism 'are organised such that they carry out some process, perform some function, or produce some end product [...]', which he calls the 'phenomenon to be explained by the mechanism' (see also Craver 2007, p. 27; Craver 2012). Along similar lines, has argued that: '[a] mechanism is sought to explain how a phenomenon is produced, how some task is carried out or how the mechanism as a whole behaves'. Thus, most mechanists seem to endorse an ontic conception of explanation when they agree 'that there is at least a sense of explanation that is ontic: that real worldly mechanisms explain their phenomena by producing them' (Illari and Williamson 2011, p. 280).

In addition, some proponents of the causal/mechanistic view have developed a semantically inspired strategy which aims to motivate further the adoption of an ontic conception of scientific explanation. For instance, this argumentative strategy can be found in both Salmon's $(1984,1989)$ and Craver's $(2007,2012)$ work on the causal/mechanistic model of scientific explanation. The underlying assumption of this strategy is that the term explanation has multiple senses and thus admits of both an ontic and an epistemic reading. That is, granting the lexical ambiguity of the term explanation, these authors claim that the process of disambiguating the term yields/reveals one sense which corresponds to the conceptualisation of scientific explanation corresponding to the ontic view. The semantic-based argument then aims to show that the so-called ontic sense of explanation is conceptually basic. For example, Craver writes that:

[Salmon] defended an ontic view, according to which explanations are objective features of the world. This idea can be brought out by considering an ambiguity in the term explanation. Sometimes explanations are text descriptions, models, or representations of any sort that are used to convey information from one person to another. [...] Other times, the term explanation refers to an objective portion of the causal structure of the world, to the set of factors that bring about or sustain a phenomenon (call them objective explanations). What explains the accident? The ice on the road, the whiskey, the argument, the tears, and the severed brake cables. There are mechanisms (the objective explanations) and there are their descriptions (explanatory texts). Objective explanations are not texts; they are full-bodied things. They are facts, not representations. They are the kinds of things that are discovered and described. There is no question of objective explanations being right or wrong, or good or bad. They just are. Objective explanations, the causes and mechanisms in the world, are the correct starting point in thinking about the criteria for evaluating explanatory texts in neuroscience. [...] Good mechanistic explanatory texts (including prototypes) are good in part because they correctly represent objective explanations. Complete explanatory texts are complete because they represent all and only the relevant portions of the causal structure of the world. Explanatory texts can be accurate enough and complete enough, depending on the pragmatic context in which the 
explanation is requested and given. Objective explanations are not variable in this way (Craver 2007, p. 27, my emphasis).

In the following section, I will focus on the starting assumption of the semanticbased argument for the ontic conception of scientific explanation, namely that the term explanation is lexically ambiguous. Whilst I agree with defenders of the ontic conception that the use of the term explanation exhibits certain systematic lexical alternations, I show that these are best conceived of as symptoms of the polysemous character of the verb to explain and its nominalisation. In addition, I claim that the linguistic process of 'meaning transfer' (Nunberg 1995) is well suited to account for the observed lexical alternations in explanatory talk. I then evaluate the consequences of this proposal for the debate between ontic and epistemic conceptions of scientific explanation.

\section{Assessing the ambiguity of explanation}

In a recent paper, Cory D. Wright (2012) has defended the epistemic conception of mechanistic explanation by challenging the idea that the term explanation is lexically ambiguous in the first place. The following considerations draw on an interesting semantic puzzle noted by Wright (2012, sec. 5) concerning certain patterns observed in the use of the verb to explain and its nominalisation. Wright (2012) claims that the solution to this puzzle consists in rejecting the lexical ambiguity hypothesis. In contrast, I argue that a more satisfactory solution shows that the term explanation does indeed display a particular form of lexical ambiguity, namely polysemy.

Generally, ambiguity is taken to be a feature of linguistic expressions which may have multiple (legitimate) senses or interpretations. As a property which marks the multiple interpretability of linguistic expressions, ambiguity differs but also relates to other similar linguistic phenomena such as indexicality, vagueness, and context sensitivity. ${ }^{5}$ Moreover, it is possible to distinguish between different sources and types of ambiguities, such as lexical, syntactic or pragmatic ambiguity. Although advocates of the ontic conception of explanation are not explicit about this issue, their argument seems to rely on the lexical variety of sense ambiguity (cf. Wright 2012; see Salmon 1984, 1989; Craver 2007, 2010). Within the class of lexical ambiguous terms, one can further distinguish between homonymous expressions and polysemous expressions. A term is polysemous if it has multiple meanings which are related to each other (e.g., book, wood, long, healthy, paper, since, etc.), and it is homonymous if its multiple meanings are unrelated (e.g., bank, bark, concrete, draw, fire, commit, etc.). As will be shown in what follows, these two types of expressions tend to behave differently under some of standard tests developed in order to check for lexical ambiguity

\footnotetext{
${ }^{5}$ The arguments discussed in the following sections do not touch directly on the question of the context sensitivity of explanatory talk. Although polysemy and context sensitivity are related linguistic phenomena, the investigation of the latter type of alternation of explanatory talk falls beyond the scope of the present paper.
} 
(cf. Zwicky and Sadock 1975). In fact, this observation seems to lie at the heart of the semantic puzzled identified in Wright's (2012) paper.

Wright (2012) remarks that the verb to explain and its nominalisation, explanation, seem to qualify as having multiple meanings since they successfully pass some of the standard tests for lexical ambiguity. However, this verdict is challenged by the observation that these expressions fail to pass other tests which seems to indicate that they are not lexically ambiguous after all. Hence, the emerging puzzle is whether the term explanation has multiple meanings and if so, how close are they related to each other.

Most of the standard tests that have been developed to check for the lexical ambiguity of certain pieces of language (discourse) depend on the presence of different interpretations and on judgments regarding the acceptability of the competing interpretations. The absurdity of meaning in this type of constructions is known as zeugma or syllepsis. For instance, a common test for lexical ambiguity is the conjunction reduction test which consists in taking two sentences that contain a purportedly ambiguous term and conjoining them by using the term only once in contexts where both meanings are encouraged. If the conjunctive sentence is sylleptic or zeugmatic, then this is taken as a mark of the ambiguity of the target term. Consider the following pairs of sentences (borrowed from Wright 2012, p. 384):

1. (a) Close your $\{$ door/eyes $\}$, and then go to sleep.

(b) Close your door and your eyes, and then go to sleep.

2. (a) The $\{$ mistakes/patients $\}$ were committed.

(b) *The mistakes and the patients were committed.

3. (a) Firing patterns at the \{neuronal/managerial $\}$ level can be irregular.

(b) *Firing patterns at the neuronal and managerial level can be irregular.

4. (a) *The neuroscientist and the chain of electrochemical events explained why the neurone fired.

(b) *The explanation of why the neurone fired was given by the neuroscientist and the chain of electrochemical events.

The targeted term in (1b) - the directive close - fails the sylleptic/conjunction reduction test and because of this is not deemed ambiguous. Things stand differently however with example (2b), were it is immediately clear that were committed is predicated of mistakes and patients in semantically incongruent senses. This makes the sentence (2b) zeugmatic which in turn indicates the lexical ambiguity of the verb to commit. Similarly, in (3b), the nominal subject firing patterns is ambiguously rendered in terms of electrochemical activation and termination of employment, which is demonstrated by the fact that the conjunctive sentence is sylleptic or zeugmatic. In addition, Wright points out that the same result obtains for the verb to explain and the noun explanation as input, in (4a) \& (4b) above. As with examples (2) and (3), the results of the conjunction reduction test seem to suggest that two different senses of explained are being invoked and thus that the term is lexically ambiguous, just as Salmon (1989) and Craver (2007) claim.

However, Wright (2012) correctly points out that there is competing evidence which goes against attributing lexical ambiguity to the term explanation. More precisely, the results of other ambiguity-detecting tests seem to contravene to the outputs 
of the syllepsis/conjunction reduction test. One such test checks for a target term with two or more unrelated antonyms. For instance, the expression light is ambiguous in English between not heavy and not dark. No similar pair of antonyms seems to be available in the case of the participle explained or cognate expressions.

Another ambiguity-detecting test is the test for contradiction, which checks whether declarative sentences containing potentially ambiguous expressions can be both truly affirmed and truly denied at the same time. If the apparent contradiction of these sorts of constructions is eliminated by the disambiguation of the target term, then the term is deemed to be ambiguous. Conversely, if the construction remains contradictory, then the target term is not lexically ambiguous.

5. (a) That bank is not a bank.

(b) *The disease was diagnosed and was not diagnosed.

(c) * To explain is to not explain. ${ }^{6}$

In (5a) we can both affirm and deny the construction, since by disambiguating the term bank, we get rid of the contradictory result. This is then taken to imply that bank is lexically ambiguous. However, in the case of examples (5b) and (5c), there does not seem to be any reasonable interpretation of the verbs to diagnose and to explain which would eliminate the apparent contradictoriness of the declarative sentences. In light of the result of this test we are again led to conclude that the term explanation is not lexically ambiguous.

Finally, Wright points out that explanation-talk fails yet another kind of test which involves the construction of syllogistic ambiguities. Consider the following syllogism (cf. Wright 2012, p. 386):

6. The bike wheels are completely true.

7. What is completely true cannot be false.

8. $\therefore$ The bike wheels cannot be false.

This syllogism involves an equivocation of its major term, because true in (6) has the meaning of being corrected so as to spin along a vertical plane, but has the meaning of maximally accurate in the minor premise (7). Wright (2012) contends that the term explanation does not give rise to similarly ambiguous syllogisms, and concludes that explanatory-talk is not lexically ambiguous after all.

At this point one might object that Wright's argument strategy merely privileges the results of certain ambiguity-detecting tests over others. This objection seems to be supported by his claim that one should not take the results of any of these tests to fix all facts about the meanings of explanation. Thus, he claims that whilst these tests 'enable understanding and insight about ambiguity, and while some test might count as (defeasible) evidence in favor of positive or negative claims about ambiguity, they are not definitive' (Wright 2012, p. 387). Wright seeks to avoid the criticism that his strategy cuts both ways by pointing out that our reliance on the results of these standard tests 'comes, in part, from statistical demonstrations of their predictive and convergent validity.' He goes on to say that '[i]t is precisely because tests for

\footnotetext{
${ }^{6}$ Examples borrowed from Wright 2012, p. 386.
} 
ambiguity do not provide absolute or definitive evidence that we appeal to a large battery of them. Were syllepsis/conjunction reduction the only test used, we might be subject to false positive results' (ibid.).

In support of his claim that the sylleptic result of applying the conjunction test to the verb to explain and its nominalisation is a false positive, Wright develops a complementary hypothesis about why these terms seem to pass the test in the first place. According to this hypothesis, the purported ontic sense of the term explanation is metaphorical (figurative), and in that sense, derivative from its epistemic interpretation. That is, Wright (2012, p. 388) contends that the following pieces of discourse are equally metaphorical:

9. (a) My car refuses to start.

(b) The mechanism of long term potentiation explains synaptic plasticity.

He claims that just as talk of cars being moody or having propositional attitudes cannot be interpreted literally, neither talk of mechanisms explaining can, or ought to be taken literally. Wright seeks to reinforce this claim by suggesting that the latter type of discourse deploys certain elliptical techniques which facilitate the metaphorical interpretation of certain types of explanatory discourse. More precisely, he claims that the following pair of sentences exploit similar elliptical linguistic techniques:

10. (a) Average people think they aren't (average).

(b) Mechanistic explanations involve (models and representations of) mechanisms. $^{7}$

If his supposition were correct, then it would seem to support the idea that the term explanation has a unique interpretation since the semantic material deleted by the elliptical mechanism (i.e., 'models and representations of') is a clear symptom of precisely the epistemic conception of explanation. Nevertheless, I think there are good reasons to reject this analogy. For instance, in (10a), the missing phonological material is nevertheless present in the surface structure of the sentence (sic), whereas (10b) does not likewise contain the purported deleted material. However, the availability of the ellipsis process requires that the first condition be met (Johnson 2008). Thus, although given a certain context, the proposed epistemic interpretation for (10b) might be perfectly legitimate, it does not seem to be fixed by any linguistic material overtly contained in the original sentence. More generally, I contend that if Wright's claim that the ontic interpretation of explanatory talk is always figurative and/or that it always exploits elliptical techniques is to carry any weight, then he must say more about the linguistic operations that are at play in these cases. Otherwise, the proposal risks to remain too superficial and ultimately unconvincing.

Thus, I conclude that Wright's (2012) proposal to solve the puzzle of the lexical ambiguity of explanatory talk by claiming that the ontic interpretation is metaphorical or figurative and that it rests on the utilisation of elliptical techniques faces serious challenges. Moreover, I claim that his argument against the lexical ambiguity

${ }^{7}$ Cf. Wright 2012, p. 388. 
of explanatory talk overlooks an important distinction between at least two different types of lexically ambiguous expressions, namely homonyms and polysemous expressions. In fact, his argument seems to rest almost entirely on the (more or less explicit) comparison between certain semantic variations exhibited by homonymous expressions and certain patterns observed in explanatory talk. ${ }^{8}$ In addition, Wright (2012) dismisses too quickly the possibility that the term explanation is polysemous (cf. Wright 2012, p. 385). In the following section, I would like to analyse precisely this hypothesis and show that it affords a better account of the lexical alternations observed in explanatory talk.

\section{Transfers of meaning in explanatory talk}

As noted above, one standard way to draw the distinction between homonymous and polysemous expressions is to say that while both categories of expressions have multiple meanings or interpretations, only the meanings of the polysemous expressions are related to each other, whereas the multiple meanings of homonyms are relatively unrelated. It is beyond the scope of this paper to provide a precise characterisation of what it is for the different meanings of an expression to be related, but in what follows I will emphasise certain candidate patterns of relations which do seem to hold between the senses of a polysemous expression. Moreover, although I take this sort of semantic relatedness to be a matter of degree, I will leave open the question of how closely do the meanings of an expression have to be in order for it to count as polysemous. However, this should not affect the validity of the solution proposed to the puzzle of the lexical ambiguity of explanation.

I start by noting that I agree with Wright's (2012) observation about the disanalogy between the results of applying the standard ambiguity-detecting tests to homonymous expressions and to explanatory talk, respectively. As shown in the previous section, the former successfully pass most (if not all) of the tests, whereas the verb to explain and its nominalisation seem to pass only the sylleptic/conjunction test, thus giving rise to the lexical ambiguity puzzle. This contrast seems largely to motivate Wright's (2012) argument that the term explanation is not lexically ambiguous at all. However, in making this claim, Wright disregards the possibility that the term explanation, whilst not a homonym, might nevertheless be polysemous.

A first observation that seems to indicate that this hypothesis is on the right track is that polysemies in general tend to yield unstable results to ambiguity-detecting tests. For instance, the term healthy seems to pass the reduction conjunction test (cf. (11a) below), just like explanation does, but not some of the other tests cited by Wright (2012). For instance, the term does not have distinct antonyms which help

\footnotetext{
${ }^{8}$ Wright (2012, p. 385 ) points out that the term explanation does not seem to be homophonic (univocal phonological structure and different orthographic and semantic structures), homographic (single orthographic form but different phonological and semantic poles), or homonymous (same orthographic and phonetic structure but constituting fully separable and unrelated lexical items).
} 
disambiguate between its two meanings, nor does it pass the contradiction test (cf. (11b)).

11. (a) The boy and the carrot are healthy.

(b) That patient was healthy and not healthy.

A similar point can be made by considering another common test for ambiguities which relies on the behaviour of expressions under anaphora. Just as in the case of the reduction conjunction test, certain anaphorical constructions that contain ambiguous terms lead to zeugmatic readings which sound infelicitous or awkward. However, even in the case of this test, there is an important disanalogy between homonyms and polysemous words, since polysemy does not always lead to zeugmaticity. Consider the following examples:

12. (a) I got up from the bank and then got some money from it.

(b) After John told me about this book, I bought it and started to read it immediately.

(c) The mechanism of the flagellum that explains the locomotion of bacteria was published in a peer-reviewed journal and it is now very influential.

(d) Tom likes to run so he goes for one every morning.

(e) Sarah hoped for change and found some in her pocket.

Whereas sentence (12a) which contains the homonym bank is clearly zeugmatic, the polysemous word book does not seem to lead to zeugmaticity in (12b). Likewise for the term mechanism in (12c). However, sentences (12d) and (12e) show that zeugmaticity is nevertheless noticeable with polysemous expressions whose meanings belong to different syntactic categories or that are intuitively less closely related. The important point here is not that the behaviour of expressions under conjunction or anaphora is an infallible test to decide in favour or against polysemy, but rather that there are certain interesting similarities between the behaviour of the term explanation and other polysemous expressions under these ambiguity-detecting tests.

Therefore, unlike Wright (2012) who takes the inconsistency of the results of these tests as a symptom of non-ambiguity, I argue that explanatory talk exhibits a certain type of lexical ambiguity, i.e., polysemy, and that the ontic and epistemic meanings of the term explanation are more closely related than typically assumed. In support of this point, I will describe in what follows a linguistic process which might be taken to be responsible for the polysemic character of explanatory talk.

More precisely, I aim to extend a proposal put forward by Geoffrey Nunberg (1995) who introduces the notion of transfer of meaning in order to characterise 'the ensemble of productive linguistic processes that enable us to use the same expression to refer to what are intuitively distinct sorts of categories of things' (Nunberg 1995, p. 109). Although the notion of transfer of meaning can be taken to cover a large variety of rhetoric devices such as metaphors, metonymies, and synecdoches, Nunberg (1995) insists that unlike rhetorical figures that 'are typically defined according to the independent conceptual relations that they exploit', transfers of meaning should be conceived of as linguistic processes which cannot be fully characterised by relying solely on conceptual analysis. Moreover, this implies that figures like metaphor or metonymy are best thought of as different sets of conditions that can support these 
processes, rather than distinct types of transfers of meaning. Thus, if the proposal sketched below is on the right track, it will provide a more direct answer to the lexical ambiguity puzzle than Wright's metaphor-based account of the alternations observed in explanatory talk.

Nunberg's (1995) paper focuses on the operation of predicate transfer which takes the name of a property that applies to certain things in one domain into a new name of a property which applies to things in another domain, provided that the two properties correspond in a particular way. More precisely, he argues that this process of predicate transfer is subject to two general conditions, which require that: (i) the first and the second property stand in a functional relation and (ii) the second property be a salient ('noteworthy') feature of the object to which it applies. In addition, Nunberg (1995) points out that the operation of predicate transfer is available for property names which are expressed by verb phrases, adjectives, but also by common nouns, whether they appear in predicate position or referring position. Thus, the operation of predicate transfer is responsible for the new meanings of the predicates and the noun phrases occurring in the following examples (borrowed from Nunberg 1995, p. 110-115):

13. (a) I am parked out back.

(b) I'm in the Whitney Museum.

(c) Who is the ham sandwich?

(d) The french fries is getting impatient. [where $I=$ my car]

$[I=$ my paintings $]$

[the ham sandwich $=$ customer]

[as above]

In these examples, the operation of predicate transfer exploits certain functional relations (correspondences) between the locations of cars in a lot and the properties of owners of these cars, between painters and some of their paintings exhibited in a certain museum, and persons in a restaurant and the dishes they have ordered. However, as noted above, Nunberg (1995) insists that the process of predicate transfer is 'only possible when the property contributed by the new predicate is "noteworthy" (cf. Nunberg 1995, p. 114), which intuitively means a property that matters. For instance, he points out that utterances like (13a) 'I am parked out back' or 'I am in the phone book', serve 'to classify the members of one set of things (e.g., garage customers) relative to immediate conversational purposes (of discharging the attendant's responsibilities, say) in virtue of their relation to the identifying properties of some other groups of things (the cars).' (ibid.). Otherwise put, this particular functional property is deemed to be 'noteworthy' because it offers a useful way of classifying its bearer relative to some particular conversational interests. The same point applies to examples (13c)-(13d). A different type of noteworthiness is exemplified by utterances like (13b) or 'American Express is in Singapore now' (which means that American Express services are available in Singapore now) where the consequences of the derived property go beyond the immediate conversational purposes. Despite their differences, both types of relations show why noteworthiness is a necessary condition for predicate transfer, and correctly predict the infelicity of an utterance like 'I was driven by Jean Gabin' (even in a context in which it might be relevant to say 'My car was once driven by Jean Gabin') or of 'I'm in the second crate on the right' uttered by a painter who might otherwise legitimately say about one of her paintings, 'I' $m$ in the Whitney Museuam' (cf. Nunberg 1995, p. 113). 
I have focused mainly on the second (i.e., noteworthiness) condition of the availability of predicate transfer because it has some interesting implications for our main topic, viz. the polysemy of explanatory talk. Note that, noteworthiness can be broadly conceived of as a special case of relevance. This suggests that there are important pragmatic conditions on the use of utterances such as (13a)-(13e). For instance, many of the examples provided above are dependent on the availability of a specialised context. However, as suggested above, that there are some cases where 'the property correspondence that licences predicate transfer holds across a wider range of situations, providing a more context-independent way of classifying the bearers of the derived properties, which answers to more general interests' (Nunberg 1995, p. 117).

Thus, on the one hand we have cases of predicate transfer where the functional relation between the two domains of things can only be exploited in a restricted range of situations. Following Nunberg (1995, p. 116), I suggest that cases such as 'the ham sandwich' are better thought of as 'occurrent metonymies', rather than polysemous expressions (i.e., having one additional lexical sense along the lines of 'ham sandwich orderer'). On the other hand, there are cases where the availability of meaning transfer for adjectives, common nouns, or verb phrases can be taken to underlie the patterns of lexical alternations associated with the phenomenon of polysemy. Nunberg (1995, p. 117) identifies several cases where the context-sensitivity of certain expressions has been reduced by their repeated usage: painters for works (a Picasso, a Derain, etc.), containers for volumes of stuff (She drank two glasses), writer for oeuvre (fifty pages of Wordsworth), place for inhabitants (Indianapolis voted for the referendum), etc. In these cases, it seems legitimate to say that the transferred predicate constitutes a new meaning of the target expression which is thereby deemed to be polysemous.

Although from one point of view there is no qualitative difference between these lexical alternations and the cases of occurrent metonymy (e.g., they are both subject to the noteworthiness conditions and to pragmatic constraints that determine their acceptability), there are also some important differences. For instance, unlike occurrent metonymies, the lexical alternations associated with polysemy tend to have a high text frequency, they become 'idiomatic' and even develop specialised senses that cannot be predicted solely on the basis of general principles of meaning transfer.

My contention is that the verb to explain and its nominalisation exhibit the latter type of lexical alternations. That is, in the case of explanatory talk, the transfer of meaning process exploits the relevant (i.e., noteworthy) correspondence between, for example, descriptions or representations of mechanisms and the complex systems they describe or represent. In other words, mechanisms acquire the new property of being explanatorily relevant (in certain appropriately circumscribed contexts) in virtue of the correspondence between the descriptions or representations of mechanisms and the mechanisms themselves, which, as noted in Section 2, are complex systems whose component parts are organised so that they produce the behaviour exhibited by the system as a whole. Moreover, the frequency of usage of the transferred reading of the term explanation (emphasised by proponents of the ontic conception of mechanistic explanation) further supports the fact that the lexical alternations observed in explanatory talk are not merely occurrent metonymies. 
Thus, I claim that the availability of the operation of predicate transfer in the case of explanatory talk supports the hypothesis that the term explanation is polysemous. That is, there are good reasons to believe that the term explanation has two closely related lexical meanings which correspond to the ontic and epistemic conceptions of explanation.

The question that remains to be addressed now is which of these two senses can be legitimately thought of as being primary and which one as being derived. In the general case of predicate transfer, where we have a sentence of the form ' $\mathrm{x}$ is $\mathrm{P}$ ', $x$ is a word that denotes something of the sort $X$, and $P$ is a predicate that ordinarily applies to a different class of things $Y$, and we have to decide whether it is $x$ or $P$ that have a transferred meaning. Likewise, if we consider the sentence:

14. The mechanism of long term potentiation is the explanation of synaptic plasticity.

two possibilities seem to be available: either mechanism has the derived meaning of mechanistic description corresponding to the epistemic conception of mechanistic explanation or explanation has the derived meaning corresponding to the ontic conception of explanation. To test this disjunction, we could further consider the following two sentences:

15. (a) The mechanism of long term potentiation that explains the phenomenon of synaptic plasticity comprises the activation of certain ion channels in the neuronal membrane.

(b) The mechanism of long term potentiation that explains synaptic plasticity can be represented by the Hodgkin-Huxley equation.

Notice that whereas (15a) encourages the ontic reading of the term explanation, (15b) promotes the epistemic reading instead. However, the fact that neither of the two sentences seems to be zeugmatic suggests that: (i) bi-directional predicate transfer is possible 9 and (ii) both senses of explanation can be felicitously used in certain specific contexts. At this point it should be noted that the operation of predicate transfer which has been taken to be responsible for the polysemic character of the term explanation does not rule out that there are additional semantic and pragmatic principles which play an essential role in producing appropriate/correct explanation-ascriptions. That is, the operation of predicate transfer by itself cannot account for the various types of context sensitivity observed in explanation-ascriptions. Moreover, the proposed solution to the ambiguity of explanation puzzle is compatible with the idea that there are other extra-linguistic factors which determine whether in a given context an ontic or an epistemic reading of explanation is appropriate. Perhaps a case could be made that the epistemic reading of explanation is conceptually primary in certain contexts, but the present argument seems to support a relatively weaker conclusion. Thus, I claim that the previous linguistic considerations cannot definitively settle the debate between ontic and epistemic conceptions of explanation, but they do provide some insight into the close connection between the two conceptions.

\footnotetext{
${ }^{9}$ For more examples of inter-definable categories which support the hypothesis of bi-directional property transfer, see Nunberg (1995, p. 124-125).
} 


\section{Concluding remarks}

One of the primary interests in discussing the case for the lexical ambiguity of the term explanation is to evaluate a more general issue, namely the import of our best linguistic theories of explanatory talk for our epistemological and metaphysical theories of (scientific) explanation. The arguments proposed in this paper suggest that for the purposes of developing a substantive and insightful analysis of the notion of explanation, these different sorts of accounts are best thought of as complementary. Thus, the idea is not to use linguistic arguments in order to rule out a competing conception of explanation. This strategy is problematic, since it would be completely fortuitous if our explanatory talk came anywhere close to determining all the relevant facts that a theory of explanation aims to account for. However, this underdetermination does not licence us to ignore the various semantic features and alternations exhibited by our explanatory talk, especially when they might suggest new ways of approaching the problem of explanation.

This paper has focused on a strategy used by some advocates of the ontic conception of scientific explanation (e.g., Salmon 1984; Craver 2007) who draw on the ambiguity of the term explanation in order to argue that the ontic sense of explanation is conceptually prior to the epistemic sense. I have shown that Wright's (2012) semantic-based argument against the ontic conception of mechanistic explanation identifies an interesting puzzle concerning the lexical ambiguity of explanatory talk but also that it ultimately fails to provide a satisfactory solution to it. In response, I have argued that explanatory talk exhibits a sort of lexical ambiguity, namely polysemy, which can be accounted for in terms of the linguistic operation of predicate transfer (cf. Nunberg 1995). In addition, I have shown why these linguistic arguments cannot, and should not be taken to decide between an ontic or an epistemic conception of scientific explanation. Rather than polarising the two positions, I have suggested that the process of bi-directional meaning transfer underlying the polysemy of explanation supports the continuity between the two philosophical projects. This conclusion, albeit modest, supports the idea that the linguistic study of explanatory talk is not only instrumentally important, and that it constitutes a source of insight and understanding of the nature of explanation.

Open Access This article is distributed under the terms of the Creative Commons Attribution 4.0 International License (http://creativecommons.org/licenses/by/4.0/), which permits unrestricted use, distribution, and reproduction in any medium, provided you give appropriate credit to the original author(s) and the source, provide a link to the Creative Commons license, and indicate if changes were made.

\section{References}

Abrahamsen, A., \& Bechtel, W. (2006). Phenomena and Mechanisms: Putting the Symbolic, Connectionist, and Dynamical Systems Debate in Broader Perspective, In Stainton, R. (Ed.) Contemporary Debates in Cognitive Science (pp. 159-187): Blackwell.

Bechtel, W., \& Richardson, R.C. (1993/2010). Discovering Complexity: Decomposition and Localization as Strategies in Scientific Research. MIT Press/Bradford Books. 
Bechtel, W. (2008). Mental mechanisms: Philosophical perspectives on cognitive neuroscience. Oxford: Routledge.

Craver, C.F. (2007). Explaining the brain: Mechanisms and the mosaic unity of neuroscience cambridge. MA: Oxford University Press.

Craver, C.F. (2012). Scientific Explanation: The Ontic Conception, In Hutteman, A., \& Kaiser, M. (Eds.) Explanation in the Biological and Historical Sciences. Berlin: Springer.

Glennan, S. (1996). Mechanisms and the nature of causation. Erkenntnis, 44(1), 49-71.

Glennan, S. (2002). Rethinking mechanistic explanation. Proceedings of the Philosophy of Science Association, 3, 342-53.

Glennan, S. (2005). Modelling mechanisms. Studies in History and Philosophy of Science Part C, 36(2), $443-64$.

Hempel, C.G., \& Oppenheim, P. (1948). Studies in the logic of explanation. Philosophy of Science, 15(2), $135-175$.

Illari, P., \& Williamson, J. (2011). Mechanisms and real and local, In McKay Illari, P., Russo, F., \& Williamson, J. (Eds.) Causallity in the Sciences: Oxford University Press.

Illari, P. (2013). Mechanistic explanation: Integrating the ontic and epistemic. Erkenntnis, 78(2), 237-255.

Johnson, K. (2008). Introduction, In Johnson, K. (Ed.) Topics in Ellipsis (pp. 1-15): Cambridge University Press.

Kim, J. (1994). Explanatory knowledge and metaphysical dependence. Philosophical Issues, 5, 51-69.

Lewis, D. (1986). Causal explanation, In Lewis, D. (Ed.) Philosophical Papers, (Vol. II pp. 214-240): Oxford University Press.

Machamer, P., Darden, L., \& Craver, C.F. (2000). Thinking about mechanisms. Philosophy of Science, $67(1), 1-25$.

Nunberg, G. (1995). Transfers of meaning. Journal of Semantics, 12(2), 109-132.

Salmon, W.C. (1984). Scientific Explanation and the Causal Structure of the World. Princeton: Princeton University Press.

Salmon, W.C. (1989). Four decades of scientific explanation, In Kitcher, P., \& Salmon, W.C. (Eds.) Scientific Explanation: University of Minnesota Press.

Sheredos, B. (2015). Re-reconciling the Epistemic and Ontic Views of Explanation. Erkenntnis. First online 30 Sept. 1-31.

van Eck, D. (2015). Reconciling ontic and epistemic constraints on mechanistic explanation, epistemically. Axiomathes, 25, 5-22.

Wright, C.D., \& Bechtel, W. (2007). Mechanisms and psychological explanation, In Thagard, P. (Ed.) Philosophy of Psychology and Cognitive Science: Elsevier.

Wright, C.D. (2012). Mechanistic explanation without the ontic conception. European Journal of Philosophy of Science, 2(3), 375-394.

Wright, C.D. (2015). The ontic conception of scientific explanation. Studies in History and Philosophy of Science Part A, 54, 20-30.

Zwicky, A., \& Sadock, J. (1975). Ambiguity tests and how to fail them. Syntax and Semantics, 4, 1-36. 